\title{
Commentary The Many Faces of MeCP2
}

\author{
Jacqueline F McGinty*,' \\ 'Deptartment of Neurosciences, Medical University of South Carolina, Charleston, SC, USA
}

Neuropsychopharmacology (2012) 37, 313-314; doi:I0.1038/npp.20II.246

Methyl-CpG-binding protein 2 (MeCP2) is an X-linked transcription factor that binds to methylated CpG dinucleotides in the genome. Its primary function was originally thought to be limited to repression of gene transcription in keeping with the transcriptional silence of methylated genes. CpG methylation is a hallmark of epigenetic regulation of genome function because it is copied to replicating DNA strands during cell division and is maintained across development as a form of epigenetic memory. Cytosine methylation in $\mathrm{CpG}$ clusters (islands) in the genome can modify chromatin structure by inhibiting activator DNA-binding proteins from binding to recognition sequences in DNA while allowing access by repressor proteins to methyl-CpP binding domains (MBD) that prevent the activation of gene transcription. MeCP2 is a member of the MBD family of proteins that associate with histone deacetylases (HDACs) and histone metyltransferases (HMTs) to form stable repressor complexes. MeCP2 point mutations cause Rett syndrome, the most common inherited form of mental retardation in girls. However, exactly which protein partners are affected by different $\mathrm{MeCP} 2$ mutations is controversial. For example, MeCP2 has been shown to associate with the Sin3a-HDAC chromatin remodeling complex in the brain-derived neurotrophic factor ( $B d n f)$ gene to modulate its expression, and loss of $\mathrm{MeCP} 2$ from the $B d n f$ promoter causes loss of local histone methylation (Chen et al, 2003; Martinowich et al, 2003). However, the function of MeCP2 may be determined by its multifaceted interactions with many different protein partners leading to different functional outcomes. Indeed, $\mathrm{MeCP} 2$ appears to associate with many different complexes in brain nuclear extracts some of which repress, but others, like CREB, activate, gene transcription (Chahrour et al, 2008).

An alternative interpretation of MeCP2's function in the brain is that it alters the global chromatin state of neurons and regulates transcriptional noise in a manner that depends on the methylation state of DNA (Skene et al, 2010 ) and the activation state of MeCP2. MeCP2 is regulated

*Correspondence: Dr JF McGinty, Deptartment of Neurosciences, Medical University of South Carolina, Charleston, SC, USA, Tel: + I 843792 9036, Fax: + I 843792 4423,

E-mail: mcginty@musc.edu

Received 13 September 20 II; accepted I3 September 201 I differentially by phosphorylation at several serine sites. Calcium calmodulin kinase-dependent phosphorylation at S421 releases MeCP2 from the $B d n f$ promoter and facilitates its transcription (Zhou et al, 2006). With regard to drugs of abuse, manipulations of MeCP2 levels in the nucleus accumbens (Nac) alter drug-induced conditioned place preference (Deng et al, 2010) and manipulations in the dorsal striatum alter escalation of cocaine intake (Im et al, 2010). Further, amphetamine (AMPH) selectively increases p-Ser421-MeCP2 in the prelimbic cortex (PLC) and in GAD67-positive GABA/parvalbumin-positive interneurons in the NAc in a D1 receptor-dependent manner (Deng et al, 2010). This expression pattern differs markedly from AMPH-induced immediate early gene or phospho-CREB induction.

In the current issue of Neuropsychopharmacology, Hutchinson et al (2011) address the mechanisms underlying this selective induction of p-Ser421-MeCP2 (pMECP2) in NAc interneurons. After pharmacologically blocking individual monoamine transporters (DAT, SERT, NET), they report that DAT inhibition by GBR 12909 increased locomotor activity and PMeCP2 in NAc interneurons and PLC, whereas SERT inhibition by citalopram decreased locomotor activity and increased pMeCP2 expression in NAc that is not restricted to $\mathrm{GABA} /$ parvalbumin interneurons. Furthermore, citalopram did not induce pMeCP2 in PLC. NET inhibition by reboxetine decreased locomotor activity but had no effect on pMeCP2 expression in NAc or PLC. The authors then determined that a D1 receptor agonist, SKF81297, induced pMeCP2 in a variety of NAc neuronal types and that a D2-selective agonist, quinpirole, by itself or in combination with SKF81297, or the 5HT2/3-preferring agonist, quipazine, limited pMeCP2 expression to GAD67positive interneurons. Furthermore, AMPH increased pMeCP2 to the same extent and in the same pattern in wild type, DAT knockout, and NET knockout mice whereas AMPH induced less pMeCP2 in SERT knockout mice than in wild-type mice. Based on all these data, the authors concluded that combined receptor stimulation by AMPH regulates $\mathrm{pMeCP} 2$.

These findings raise the question as to why the patterns of induction of pMeCP2 triggered by AMPH, D1, D2, or 5HT2/ 3 receptor stimulation are not similar to immediate early genes like Fos or phospho-proteins like CREB. A clue is provided by the intracellular cascades that regulate these 
proteins. Unlike Fos or CREB, MeCP2 is not a direct substrate for the adenylyl cyclase/cAMP/protein kinase A (PKA) cascade. However, forskolin-induced adenylyl cyclase in vivo caused widespread Fos and pMeCP2 expression in medium spiny neurons (MSNs) in NAc whereas forskolin induced pCREB, but not pMeCP2, in primary striatal cultures. Considering that NMDA and membrane depolarization induce pMeCP2 in striatal cultures, these data suggest that postsynaptic stimulation of MSNs is not sufficient to induce pMeCP and that in vivo forskolin may be activating presynaptic release of neurotransmitters. The authors suggest that neurotransmitter receptors that increase $\mathrm{Ca}^{2+}$ influx or release it from intracellular stores may have a more direct role in $\mathrm{MeCP}$ phosphorylation than PKA because CAM kinase II and IV phosphorylates MeCP2 (Zhou et al, 2006). In that context, it would be interesting to investigate whether stimulation of

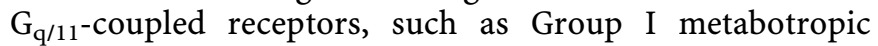
receptors, induces the same pattern of pMeCP2 as observed after D1/D2 and 5HT2/3 receptor stimulation. Finally, it remains a question as to why presynaptic D2 receptors on corticostriatal terminals activated by AMPH do not prevent pCREB induction in MSNs in vivo. Thus, the intracellular signaling events that restrict $\mathrm{pMeCP} 2$ expression to GABA/ parvalbumin neurons and the functional significance of this intriguing pattern still remain to be elucidated.

\section{DISCLOSURE}

The author declares no conflict of interest.

\section{REFERENCES}

Chahrour M, Jung SY, Shaw C, Zhou X, Wong STC, Qin J et al (2008). MeCP2, a key contributor to neurological disease, activates and represses transcription. Science 320: 1224-1229.

Chen WG, Chang Q, Lin Y, Meissner A, West AE, Griffith EC et al (2003). Derepression of BDNF transcription involves calcium-dependent phosphorylation of MeCP2. Science 302: 885-889.

Deng JV, Rodruiguez RM, Hutchinson AN, Kim I-H, Wetsel WC, West AE (2010). MeCP2 in the nucleus accumbens contributes to neural and behavioral responses to psychostimulants. Nature Neurosci 9: 1128-1138.

Hutchinson AN, Deng JV, Aryal DK, Wetsel WC, West AE (2011). Differential regulation of $\mathrm{MeCP} 2$ phosphorylation in the CNS by dopamine and serotonin. Neuropsychopharmacology 37: 321-337.

Im H-I, Hollander JA, Bali P, Kenny PJ (2010). MeCP2 controls BDNF expression and cocaine intake through homeostatic interactions with microRNA-212. Nature Neurosci 13: 1120-1127.

Martinowich K, Hattori D, Wu H, Fouse S, He F, Hu Y et al (2003). DNA methylation-related chromatin remodeling in activitydependent Bdnf gene regulation. Science 302: 890-893.

Skene PJ, Illingworth RS, Webb S, Kerr ARW, James KD, Turner $\mathrm{DJ}$ et al (2010). Neuronal MeCP2 Is expressed at near histoneoctamer levels and globally alters the chromatin state. Mol Cell 37: 457-468.

Zhou Z, Hong EJ, Cohen S, Zhao WN, Ho HY, Schmidt L et al (2006). Brain-specific phosphorylation of MeCP2 regulates activity-dependent Bdnf transcription, dendritic growth, and spine maturation. Neuron 52: 255-269. 